\title{
SISCOM: Cooperative Multi-Robot Systems in Construction
}

XXIV International Conference of the Iberoamerican Society of Digital Graphics Medellin | Colombia

\author{
Luis Felipe González-Böhme \\ Universidad Técnica Federico Santa María | Chile | luisfelipe.gonzalez@usm.cl \\ Rodrigo García-Alvarado \\ Universidad del Bío-Bío | Chile | rgarcia@ubiobio.cl \\ Francisco Javier Quitral-Zapata \\ Universidad Técnica Federico Santa María | Chile | francisco.quitral@usm.cl \\ Eduardo Antonio Valenzuela-Astudillo \\ Universidad Técnica Federico Santa María | Chile | eduardo.valenzuelaa@usm.cl
}

\begin{abstract}
We present an ongoing research project focused on the development of more efficient setups for cooperative multi-robot systems in 3D-printed construction. Early kinematic simulations of a mobile robotic cell prototype with two ceiling-mounted orbiting manipulators have provided new insights into 3D printing topology. An extrusion nozzle is mounted on each collaborative robot whose primary function is to match the extrusion path to the print contour while they move along a circular path. The challenge of setting up on site a semi-structured environment for cooperative multi-robot 3D printing led us to think up a new species of construction 3D printer.
\end{abstract}

Keywords: 3D-Printed construction; Cooperative multi-robot system; Mobile robotic cell; Collaborative robot; Robots in architecture.

\section{INTRODUCCIÓN}

La construcción impresa, también conocida como construcción mediante impresión 3D, consiste en la fabricación aditiva de edificios y componentes constructivos mediante la deposición mecánica controlada por computador de filamentos de un mortero de material en estado plástico -generalmente con alto contenido de cemento, granos finos de áridos, usualmente entre 2 y 3 $\mathrm{mm}$ de diámetro, acelerantes y otros aditivos específicos-, que reproducen el contorno de la pieza a imprimir, en su extensión horizontal y vertical, en capas sucesivas superpuestas que se adhieren entre sí consecutivamente, para formar un continuo resistente que se solidifica progresivamente, conservando su forma y posición, sin la ayuda de encofrado (Perkins and Skitmore, 2015; Ghaffar et al, 2018; Ma et al, 2018).

Durante el fraguado inicial, el umbral de tiempo en que cada filamento de mortero de cemento se adhiere mejor al filamento inferior, en capas sucesivas superpuestas, sin aplastarse demasiado mutuamente, ni volcarse o desmoronarse, es un parámetro crucial en la programación y control de las velocidades y aceleraciones de deposición y bombeo del mortero, especialmente en la impresión de piezas de gran extensión horizontal 0 de recintos (Kazemian, 2017). Naturalmente, la composición del mortero, el número de capas superpuestas y su respectivo peso, también son factores determinantes en la programación y control del proceso de impresión 3D con mortero de cemento. Menos frecuente, hasta ahora, es la construcción impresa con mortero de materiales polímeros, biomateriales (Véliz-Reyes et al., 2019) y otros materiales compuestos.

En la construcción impresa de contornos de geometría compleja, la forma y la orientación de la boquilla por donde se extruye el mortero, también son fundamentales para determinar el alcance efectivo de la herramienta, especialmente si la pieza a imprimir contiene una armadura o ductos preinstalados, igualmente si el ángulo que los muros de la pieza forman con el suelo es distinto de noventa grados $y$, en algunos casos, también si se busca hacer más expedita la salida del filamento de mortero desde la boquilla, disminuyendo la fricción producida por la orientación vertical de la boquilla.

El proceso de construcción impresa puede ocurrir en obra, es decir en el sitio de construcción, para fabricar edificios en su emplazamiento definitivo o en taller, para prefabricar componentes constructivos que finalmente serán puestos en servicio en un lugar distinto de donde fueron impresos.

El modo convencional de fabricar muros, columnas, componentes de losas y otras piezas mediante impresión $3 D$, es imprimir de abajo hacia arriba, capa por capa, el contorno de la pieza con un filamento de mortero continuo y el dintorno de la pieza con otro o el mismo filamento, para formar una trama estructuradora de la pieza. Por ejemplo, los componentes de losas se pueden prefabricar, preferentemente en taller, imprimiéndolos en posición 
vertical, como si se tratara de muros o ladrillos huecos que finalmente son abatidos para ponerlos en servicio, tendidos en su posición y orientación definitivas. También se puede utilizar el contorno impreso de la pieza para que éste actúe como un encofrado y una vez que sus paredes endurezcan y adquieran la resistencia suficiente, rellenar el interior de la pieza con el mismo u otro material apropiado para mejorar su resistencia mecánica, aislación acústica o aislación térmica. Tanto el mortero del contorno como el del dintorno pueden contener además fibras naturales o sintéticas para mejorar su resistencia mecánica. En cualquier caso, también se pueden instalar ductos de suministro y extracción de agua, electricidad, gases y otros medios, antes, durante o después de fabricar la pieza, según lo permita el sistema de construcción impresa que se emplee. La misma condición rige para instalar alfeizares y dinteles, por ejemplo, para conformar los vanos de puertas y ventanas, antes o durante el proceso de impresión 3D de los muros que componen un recinto. En ciertos casos es necesario instalar una armadura de barras o mallas de acero en el interior de la pieza, con el fin de mejorar su resistencia mecánica, especialmente a las fuerzas laterales producidas, por ejemplo, por un sismo. Para ese fin, la armadura de la pieza debe quedar anclada al cimiento, así como a las piezas adyacentes si las hubiere, con el fin de obtener un continuo resistente, sólidamente fundado en el suelo y solidario con el resto del edificio. En ese caso es crítico planificar en detalle la forma y el orden cronológico en que se imprimirá la pieza y se instalará dicha armadura. Igualmente, la elección del sistema de construcción impresa que se emplee adquiere vital importancia, especialmente si no va a ser posible modificar la posición o la orientación de la pieza durante el proceso de impresión $3 \mathrm{D}$, como ocurre generalmente en la construcción impresa en obra.

\section{SISTEMAS DE CONSTRUCCIÓN IMPRESA}

En general, se pueden distinguir cuatro clases de sistema de construcción impresa según su estructura mecánica y volumen de impresión o construcción: cartesiano, cilíndrico, paralelo y articulado.

El sistema cartesiano de construcción impresa está compuesto fundamentalmente por un pórtico. Los eslabones de su cadena cinemática se conectan mediante, al menos, tres uniones prismáticas (traslacionales) orientadas cada una de ellas en una de las direcciones de los ejes $\mathrm{X}, \mathrm{Y}$ y $\mathrm{Z}$ del sistema de coordenadas cartesiano. Su volumen de impresión tiene la forma de un prisma rectangular (ortoedro) y está contenido completamente por la estructura portante del sistema de construcción impresa propiamente tal. La boquilla convencional por donde se extruye el material se desplaza con tres grados de libertad y con una única orientación fija. Entre los sistemas cartesianos, sobresalen "Contour Crafting" de Khoshnevis et al. (2001), "Freeform Construction" también conocido como "Concrete Printing" (Buswell et al., 2007; Lim et al., 2012), COBOD (2012) y "D-shape" (Cesaretti et al., 2014; Dini 2019) (Figura 1).

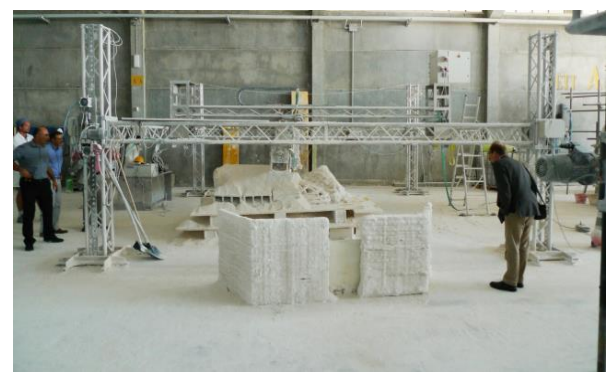

Figura 1: Sistema cartesiano de construcción impresa (D-shape, 2020).

El sistema cilíndrico de construcción impresa está compuesto fundamentalmente por un brazo giratorio voladizo. Los eslabones de su cadena cinemática se conectan mediante una unión de revolución (rotacional) en torno al eje vertical Z, una unión prismática (traslacional) también en la dirección del eje vertical $Z$ y una unión prismática (traslacional) en la dirección de uno de los ejes horizontales $\mathrm{X}$ o $\mathrm{Y}$ del sistema de coordenadas cartesiano. Su volumen de impresión adopta la forma de un cilindro incompleto -si la unión de revolución en torno al eje vertical $Z$ no alcanza los 360 grados angulares-o completo -si la unión de revolución en torno al eje vertical $Z$ si alcanza o supera los 360 grados angulares-, que contiene parcial o totalmente al sistema de construcción impresa propiamente tal. La boquilla convencional por donde se extruye el material se desplaza con tres grados de libertad y con una única orientación fija. Entre los sistemas cilíndricos, sobresale "Crane" de WASP (2018) (Figura 2).

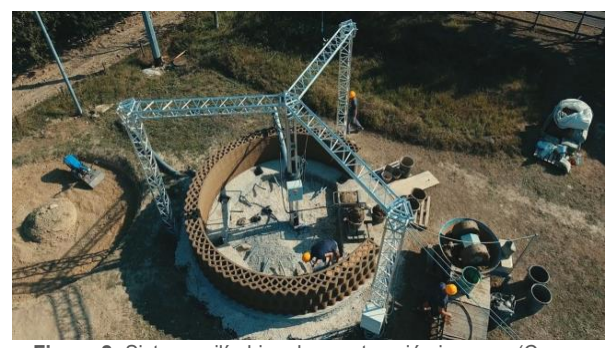

Figura 2: Sistema cilíndrico de construcción impresa (Crane 2019).

El sistema paralelo de construcción impresa, también conocido como Delta, está compuesto fundamentalmente por tres brazos articulados concurrentes o cables en tensión concurrentes. Los eslabones de la cadena cinemática de cada brazo se conectan mediante, ya sea una unión prismática (traslacional) en la dirección del eje vertical $Z$, o una unión de revolución (rotacional) en torno a uno de los ejes horizontales $X \circ Y$ y dos uniones universales (rotacionales) en torno a uno de los ejes horizontales $\mathrm{X}$ o $\mathrm{Y}$ y en torno al eje vertical $\mathrm{Z}$. Su volumen de impresión describe la forma aproximada del hemisferio inferior de una esfera o de un paraguas invertido y está contenido completamente por la estructura portante del sistema de construcción impresa propiamente tal. La boquilla convencional por donde se extruye el material se desplaza con tres grados de libertad y con una única orientación fija. Entre los sistemas paralelos, sobresalen "C4 robot" de Bosscher et al. (2007), Barnett (2015) y "BigDelta" de WASP (2015) (Figura 3). 


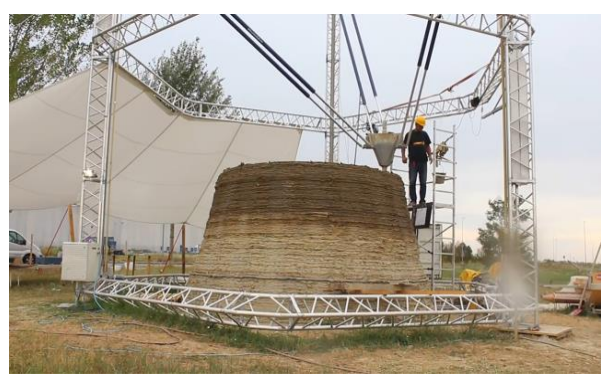

Figura 3: Sistema paralelo de construcción impresa (Big Delta, 2017).

El sistema articulado de construcción impresa está compuesto fundamentalmente por un robot manipulador. Los eslabones de su cadena cinemática se conectan mediante seis uniones de revolución (rotacionales), cada una en torno a uno de los ejes $\mathrm{X}, \mathrm{Y}$ o $\mathrm{Z}$ del sistema de coordenadas cartesiano. Su volumen de impresión se aproxima a la forma de una esfera incompleta o completa, que contiene ya sea parcial o totalmente al sistema de construcción impresa propiamente tal. La boquilla convencional por donde se extruye el material se desplaza con tres grados de libertad y se orienta con tres grados de libertad. Entre los sistemas articulados, sobresale CyBe (2012) (Figura 4).

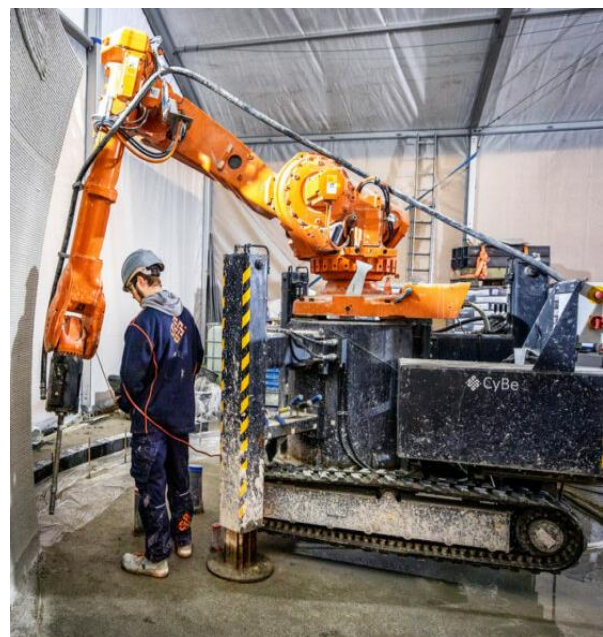

Figura 4: Sistema articulado de construcción impresa (CyBe, 2020)

En general, los sistemas cartesianos y paralelos de construcción impresa ocupan más espacio para su instalación y operación que los sistemas cilíndricos y articulados, principalmente debido a la necesidad de instalar en obra sistemas de apoyo más grandes y robustos y a veces también sistemas de guiado adicionales. Los sistemas cilíndricos y articulados de construcción impresa, aunque generalmente son autoportantes, sólo pueden imprimir a su alrededor, a menos que se instalen en obra sistemas de apoyo y guiado adicionales que les permitan desplazarse horizontalmente o verticalmente. Sin embargo, los sistemas cilíndricos y articulados no pueden imprimir su entorno completamente, sin quedar encerrados dentro de su propia obra impresa.
En todo caso, la necesidad de instalar en obra sistemas de guiado adicionales para el desplazamiento horizontal o vertical de un sistema de construcción impresa, limita las posibilidades del sistema de operar en modo enjambre. Ninguno de estos sistemas es apto para imprimir componentes constructivos verticales con armadura instalada en obra. Las barras y mallas de armadura de muros y columnas colisionarían con la viga del sistema cartesiano, el brazo radial del sistema cilíndrico, los brazos concurrentes del sistema paralelo y el brazo del sistema articulado -a menos que pueda desplazarse alrededor de dicha armadura. Esta desventaja limita significativamente el uso potencial de estos sistemas en países sísmicos. En cualquier caso, el objetivo común a todos los sistemas de construcción impresa es deponer un filamento continuo del material evitando cortes o costuras (Buswell, 2018) y minimizar el tiempo total de construcción (Kazemian et al., 2017).

\section{SISTEMA COOPERATIVO MULTI- ROBOT DE CONSTRUCCIÓN IMPRESA}

Nuestra investigación se centra en el desarrollo de nuevas configuraciones de sistemas cooperativos multi-robot para la construcción impresa en obra, que sean más eficientes desde el punto de vista de la topología de impresión 3D de recintos y componentes constructivos. Nuestros objetivos de eficiencia -no necesariamente en el orden descrito a continuación- son que el sistema permita:

- Ajustar la velocidad de deposición de las capas a umbral de tiempo de mejor adherencia y menor aplastamiento entre filamentos.

- Orientar las boquillas de extrusión en tres grados de libertad, para sortear barras de armadura, sacar el mejor provecho de su forma o facilitar la salida del mortero.

- Instalarse en obra para imprimir recintos en su emplazamiento definitivo.

- Maniobrabilidad para reubicarse en recintos.

- Prescindir de sistemas de guiado o estructuras adicionales para su montaje y operación.

- Imprimir muros con barras o mallas de armadura instaladas en obra.

- Evitar cortes o costuras en los filamentos.

El desafío de configurar en obra un entorno semiestructurado movible, que facilite la programación de una impresión 3D cooperativa multi-robot, nos condujo a pensar en una nueva especie de impresora $3 \mathrm{D}$ para la fabricación aditiva de edificios.

Simulaciones iniciales de la cinemática de un prototipo de celda robótica móvil con dos robots colaborativos orbitales, montados en techo y alimentados externamente con mortero de cemento (patente en trámite en varios países) (Figura 5) nos han brindado nuevos conocimientos sobre la topología de impresión $3 \mathrm{D}$ de recintos y componentes constructivos.

Con una boquilla de extrusión montada en cada uno, la función principal de ambos manipuladores es reproducir la trayectoria de extrusión de un contorno y dintorno diseñados previamente, al mismo tiempo que se desplazan en movimiento circular, deponiendo capas sucesivas superpuestas de filamentos continuos de un mortero de cemento. 


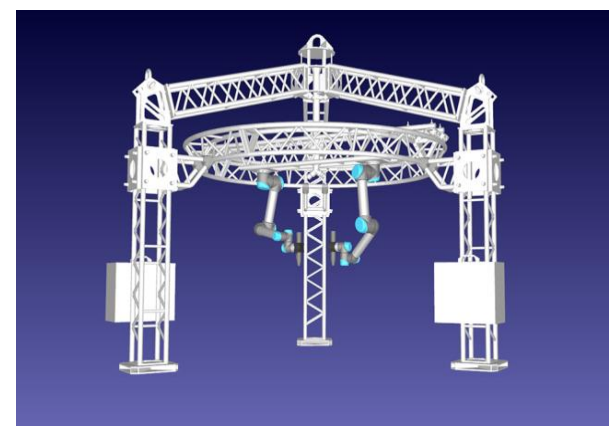

Figura 5: Modelo 3D del sistema cooperativo multi-robot,

SISCOM (patente en trámite en varios países).

\section{SIMULACIONES}

A partir de la hipótesis de impresión colaborativa con trayectorias helicoidales y en función del modelo prototipo expuesto (Figura 5), se desarrolla una réplica del mecanismo en el software RoboDK, un simulador para robots industriales y herramienta para la programación offline de trayectorias de robots, compatible con el controlador de robots colaborativos UR5/CB3, y que permite, por tanto, llevar la programación directamente a ejecución en la unidad de control, luego del desarrollo de las simulaciones.

En primera instancia los modelos tridimensionales de la estructura son importados y dispuestos en el entorno de trabajo de RoboDK, para luego establecer sistemas de referencia adecuados para la fijación y control de los robots de manera invertida en la viga giratoria. Posteriormente, mediante programación offline se desarrolla un algoritmo que establece las instrucciones operativas del sistema. Por una parte, la rotación de la viga a velocidad constante, y por otra parte, las trayectorias de desplazamiento del efector final o herramienta en cada robot. De este modo, la secuencia coordinada de estos 3 sub-mecanismos (viga giratoria, robot 1, robot 2), ejecutada desde la programación desarrollada, permite la impresión colaborativa helicoidal.

La primera figura simulada corresponde a un sólido de revolución, vale decir, una figura tridimensional que se genera mediante la rotación de una superficie plana alrededor de un eje axial o eje de revolución; un tubo de $150 \mathrm{~cm}$ de alto, correspondiente a la impresión 3D del contorno de una columna cilíndrica de $30 \mathrm{~cm}$ de diámetro rodeando la armadura (Figura 6 ).

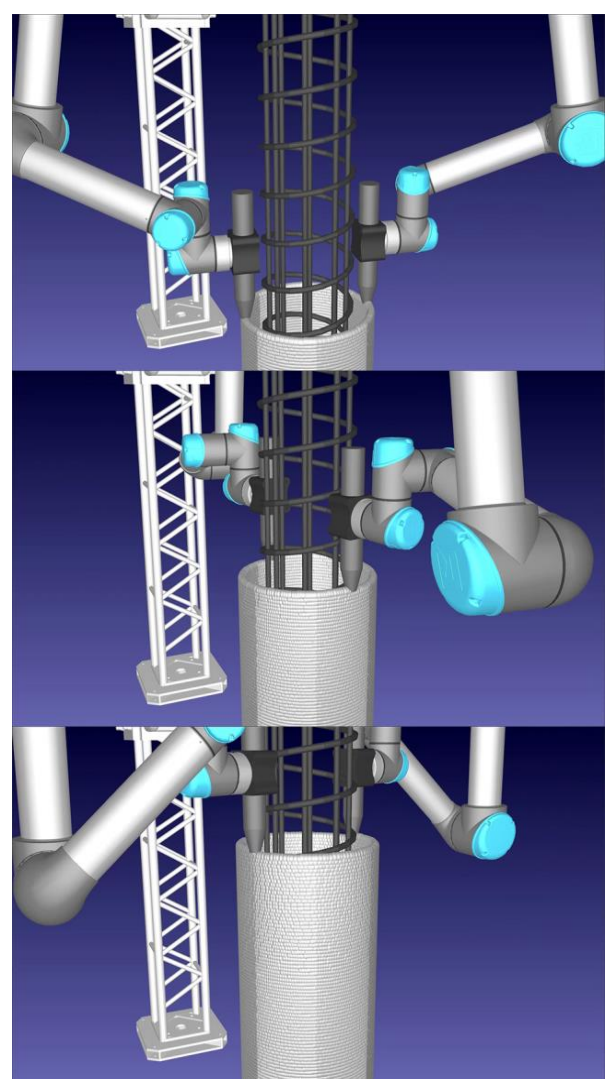

Figura 6: Simulación de la impresión 3D de una columna en concreto con armadura de refuerzo preinstalada en su interior.

El desplazamiento de cada herramienta (boquilla de extrusión), se realiza en una trayectoria vertical ascendente, la que, sumada a la rotación de la viga, permite la deposición continua en una trayectoria helicoidal, donde el parámetro de velocidad de desplazamiento de la herramienta, en este caso constante, se relaciona directamente con la altura de capa y se vislumbra como uno de los parámetros relevantes a controlar en la secuencia de impresión 3D.

Siguiendo la lógica de la viga giratoria a velocidad constante, se experimenta respecto a cómo la modificación de esta trayectoria ascendente en un plano vertical asociado al sistema de coordenadas de la base de cada robot (International Organization for Standardization, 2013), permite la impresión de diversos tipos de sólidos de revolución (Figura 7). 


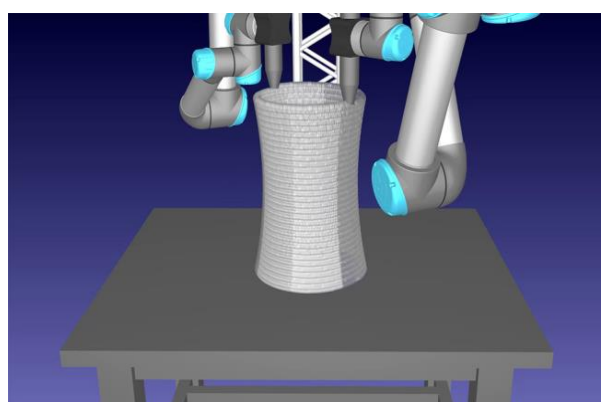

Figura 7: Simulación de la impresión 3D, variante de sólido de revolución.

Se experimenta, además, con distintos métodos para la definición de estas trayectorias de extrusión en este mecanismo, particularmente para geometrías que no correspondan a un tipo de sólido de revolución, como un tubo de sección elíptica (Figura 8).

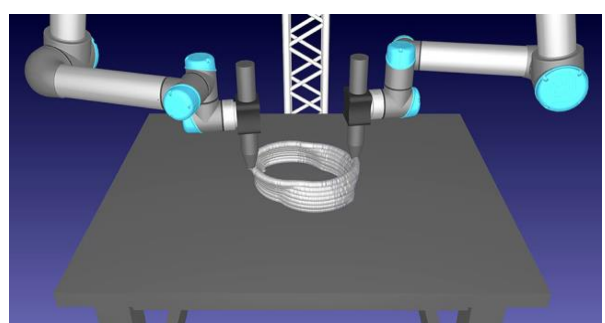

Figura 8: Simulación de la impresión 3D, tubo elíptico.

En este caso, se aplica una aproximación geométrica respecto de las posiciones de la base de cada robot, (determinadas por la rotación a velocidad constante de la viga que los sujeta) evaluadas en distintos instantes, para la definición de las trayectorias de cada herramienta en consideración de las distancias al eje de rotación de la viga, requeridas en cada instante evaluado, para el trazado de la geometría objetivo, determinando una velocidad constante y aproximada de avance de la herramienta.

Considerando que una trayectoria en línea recta vertical permite definir filamentos helicoidales para un tubo de sección circular, se intenta resolver con qué trayectorias se podría lograr la sección elíptica en la geometría. La simulación ejecutada permite determinar que esta aproximación geométrica de las trayectorias logra asemejarse a la geometría, aunque con evidentes errores, como el desfase capa a capa y la deformación de la sección elíptica. Se detecta, en consecuencia, la necesidad de una velocidad variable de avance en las trayectorias de las herramientas de cada robot, para coordinar su posición en el trazado deseado (objetivo).

Otra simulación realizada, esta vez para un tubo de sección cuadrada (Figura 9) reafirma las sospechas respecto de los requerimientos de cálculo y aplicación de velocidades y/o aceleraciones.

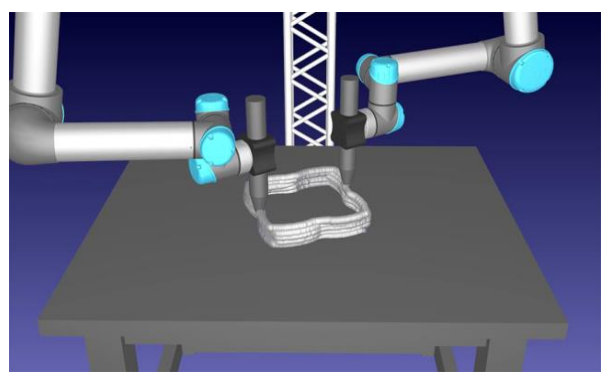

Figura 9: Simulación de la impresión 3D de un tubo de sección cuadrada.

Se aproxima, con errores, a un cuadrado en la sección transversal del tubo, evidenciando que la resolución de una figura de esas características requiere de velocidades variables en la trayectoria de la herramienta, condición que resulta aplicable en futuros desarrollos, bajo la misma lógica: cálculos de velocidad y aceleración para alcanzar la posición y orientación de la herramienta requeridas en cada tramo (aplicando criterio de resolución aceptable) en dependencia de la velocidad de rotación de la viga giratoria, para cada geometría a imprimir.

\section{IMPLEMENTACIÓN DE UN PROTOTIPO A ESCALA REAL}

Con el objetivo de establecer un banco de pruebas para la experimentación física de construcción impresa con dos robots UR5/CB3, se encuentra en desarrollo y montaje una plataforma específicamente adaptada para uso experimental en laboratorio, correspondiente a un recinto de $5 \times 4.4 \times 2.4 \mathrm{~m}$ (Figura 10).

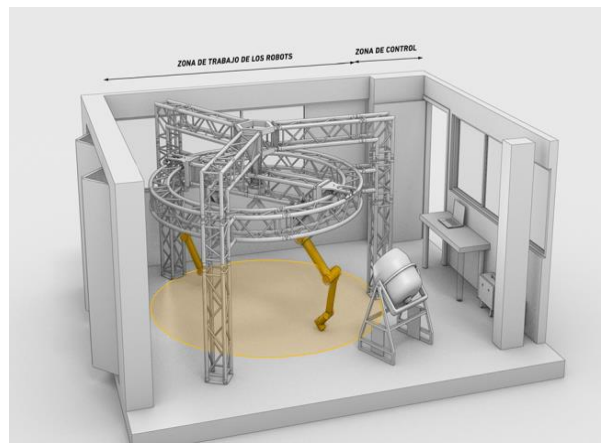

Figura 10: Modelo del laboratorio SISCOM.

De acuerdo con estas restricciones volumétricas este prototipo no implementa un movimiento vertical de los soportes estructurales o del riel circular que soporta la viga giratoria, por lo que se limita al alcance del robot, correspondiente a 1.7 metros de diámetro. 


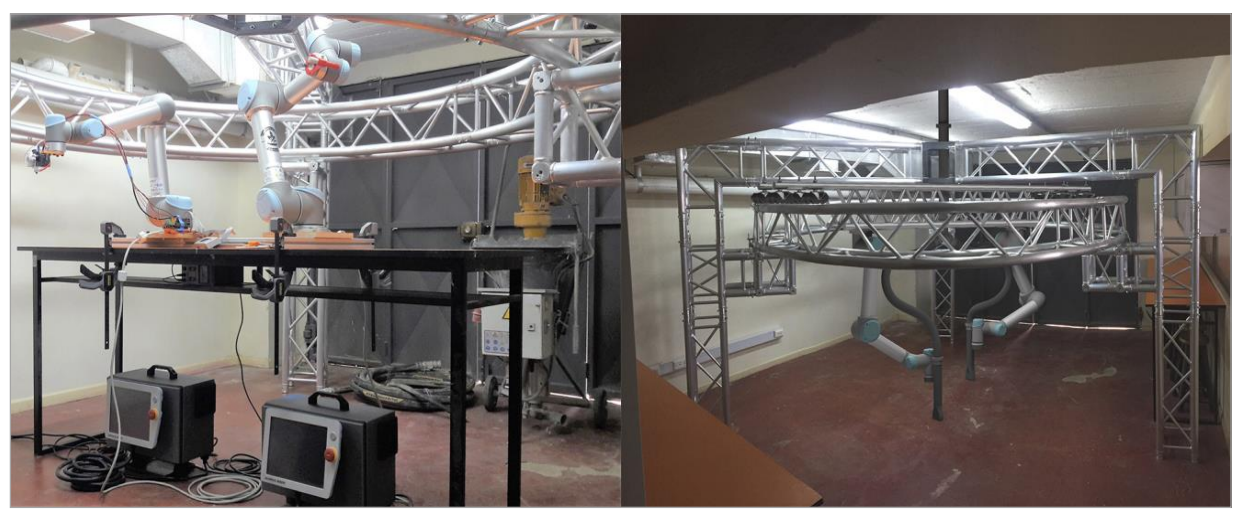

Figura 11: Prototipo del sistema cooperativo multi-robot, SISCOM, UTFSM, Valparaíso, Chile. Estado actual (izquierda), estado objetivo (derecha).

La estructura se desarrolla a partir de un sistema estructural modular multipropósito de vigas de celosía, ya instalado en el laboratorio, incluyendo el riel circular, quedando pendiente la construcción y montaje de la viga giratoria, así como el sistema de alimentación multi-robot para mortero de cemento (Figura 11).

Para la puesta a prueba de la deposición de filamentos de mortero se dispone de una bomba de mortero Putzmeister modelo P12 Sprayboy, equipo que permite la mezcla, bombeo y proyección de morteros con un tamaño de árido de $4 \mathrm{~mm}$, así como una distancia de transporte de hasta $30 \mathrm{~m}$ de longitud y $15 \mathrm{~m}$ de altura (Putzmeister, 2020).

Parte de los resultados esperados, de la construcción y puesta en funcionamiento de este prototipo, es la validación mediante una prueba de concepto y la experimentación de construcción impresa a escala, atendiendo diversos objetivos de la impresión 3D, como la correcta adherencia entre filamentos y el correcto desarrollo geométrico de los modelos a imprimir.

\section{APLICACIONES ARQUITECTÓNICAS}

La construcción se caracteriza por ser una actividad de gran relevancia social y productiva, representando cerca del $10 \%$ de la economía, con un crecimiento constante, destinado a generar viviendas, edificios e infraestructura (CChc, 2018). Sin embargo, presentan una diversidad de configuraciones y localizaciones y procesos de ejecución, que dificultan su industrialización y productividad (Corfo \& PMG, 2016). Por lo cual esta estrategia de construcción impresa 3D mediante un sistema móvil, pretende cubrir la versatilidad y dispersión de las obras a ejecutar. El sistema propuesto posee la virtud de adaptarse a diferentes condiciones y ambientes de trabajo, con una unidad de manejo múltiple, que permite realizar distintas faenas. Por ejemplo, realizar la impresión perimetral de hormigonado en torno a una armadura para elementos estructurales, logrando una ejecución continua de la forma. Como también combinar labores de armado e impresión, para la integración de refuerzos, servicios o accesorios. Trabajando en distintos sectores y alturas de la construcción de acuerdo con el grado de avance y disponibilidad de materiales. También permite complementar de manera variable durante la obra con faenas convencionales o modificaciones de procesos, o incluso de elementos, debido a su carácter móvil y múltiple.

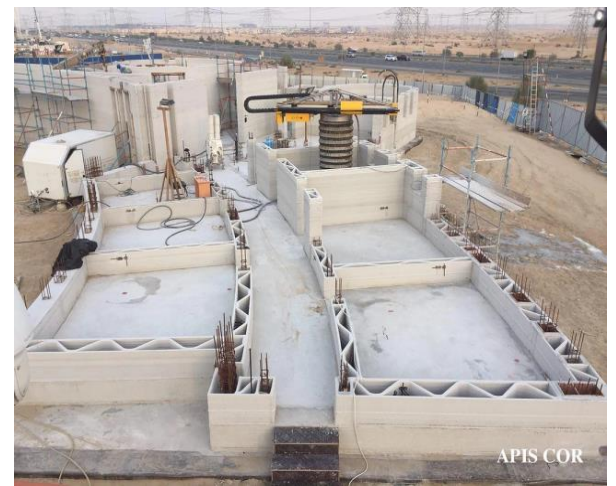

Figura 12: Municipio de Dubái, edificio impreso en 3D más grande del mundo (Apis Cor, 2019).

De modo, que el sistema propuesto plantea un enfoque de industrialización 4.0 apropiada para la construcción en terreno, que difiere radicalmente de la fábrica que puede contar con un ambiente fijo de producción. La construcción se enfrenta a ubicaciones diferentes en cada obra, en distintos tiempos y geometría diversas. Las estrategias planteadas previamente de construcción impresa-3d se enfocan a edificaciones pequeñas ejecutadas totalmente con una sola máquina, y por tanto dependientes de su tamaño, ubicación y tecnología. Mientras el sistema cooperativo multi-robot puede actuar en una diversidad de faenas y situaciones. Esta versatilidad descansa en una gestión centralizada de la obra, a partir de su modelación tridimensional y planificación de faenas, que permite orientar su utilización en los aspectos que requieren su ejecución más crítica y segura, acelerando procesos en diferentes horarios o condiciones. La productividad de la construcción se orienta a la reducción de tiempo y recursos involucrados en la ejecución apropiada para cumplir los desempeños requeridos. Por esta razón, la versatilidad del sistema de impresión es crucial para asegurar el logro de estos alcances en un campo variable de obras y actividades. 


\section{CONCLUSIONES}

El sistema cooperativo multi-robot planteado en este artículo constituye en una nueva especie tecnológica de construcción impresa 3D, que amplía sustancialmente las capacidades de esta nueva estrategia de edificación. Las simulaciones desarrolladas con este mecanismo, considerando la rotación de la viga que sujeta los manipuladores y que gira a una velocidad angular constante, permiten determinar que es posible, en el estado actual, la impresión colaborativa de cualquier tipo de sólido de revolución con filamentos continuos, vale decir, sin costuras o interrupciones y con la capacidad de rodear sistemas de armaduras de refuerzo en el caso de pilares de hormigón armado, condición imposible con un solo robot manipulador con base estática (es decir, sin ejes auxiliares).

Las pruebas realizadas para la fabricación de geometrías que no corresponden al tipo sólido de revolución, como es el caso de formas tubulares de sección elíptica o cuadrada, permiten determinar que se requiere que las trayectorias programadas para los robots se ejecuten a velocidades no uniformes, lo que implica un cálculo de velocidades y aceleraciones atingente a cada tipo de trayectoria o contorno, la cual se vislumbra viable y posible de parametrizar e implementar en un desarrollo futuro. Además, se comprueba que es posible la impresión de geometrías asimétricas, donde, de igual modo, resulta necesario realizar cálculos de velocidad y aceleración que permitan ejecutar el trazado requerido en cada caso, con una fidelidad y resolución aceptables.

La configuración propuesta puede tener implicancias revolucionarias en la construcción, al proveer un equipamiento modular y variable que se puede instalar en obra para ejecutar edificaciones en una fracción del tiempo y una reducción sustancial de materiales, personal y gestión que la construcción tradicional. Planteando un nuevo enfoque para la arquitectura conectada en terreno con la construcción automatizada para optimizar las formas y procesos de ejecución. También permite especialmente construir a distancia en zonas de riesgo, lugares remotos o extra-planetarios con una coordinación y precisión adecuada a los distintos contextos. Se requiere experimentación de materiales y combinación de tareas para asegurar la ejecución.

En futuros desarrollos se establecerán los algoritmos necesarios para la resolución de los cálculos mencionados anteriormente y su consecuente implementación en la programación actual dentro del software RoboDK, ampliando las capacidades de este sistema cooperativo multi-robot de construcción Impresa, que constituye una nueva especie de impresora 3D.

\section{AGRADECIMIENTOS}

Este proyecto es financiado por el Estado de Chile a través del Fondo Nacional de Desarrollo Científico y Tecnológico (FONDECYT 11810158).

\section{REFERENCIAS}

Apis Cor. (2019). Apis Cor 3D printed in Dubai. Retrieved October 26, 2020, from https://www.apis-cor.com/dubai-project

Barnett, E., \& Gosselin, C. (2015). Large-scale 3D printing with a cable-suspended robot. Additive Manufacturing, 7(2015), 2744.

Big Delta. (2017). Retrieved 26 October 2020, from https://www.3dwasp.com/en/giant-3d-printer-bigdelta-wasp$12 \mathrm{mt} /$

Bosscher, P., li, R. L. W., Bryson, L. S., \& Castro-Lacouture, D. (2007). Cable-suspended robotic contour crafting system. Automation in Construction, 17(1), 45-55.

Buswell, R. A., Soar, R. C., Gibb, A. G. F. \& Thorpe, A. (2007) Freeform Construction: Mega-scale Rapid Manufacturing for construction. Automation in Construction, 16, 2, 224-231.

Buswell, R., Leal de Silva, W., Jones, S., \& Dirrenberger, J. (2018). 3D printing using concrete extrusion: A roadmap for research. Cement and Concrete Research, 112, 37-49.

CChC. (2018). Informe MACh 48 - Macroeconomía y Construcción. Retrieved from https://www.cchc.cl/centro-deinformacion/publicaciones/publicaciones-mach/informemach-48

Cesaretti, G., Dini, E., De Kestelier, X., Colla, V., \& Pambaguian, L. (2014). Building components for an outpost on the Lunar soil by means of a novel 3D printing technology. Acta Astronautica, 93, 430-450.

COBOD. (2012). BOD2: Modular 3D construction printer. Retrieved July, 2020, from https://cobod.com/bod2

CORFO \& PMG. (2016). Hoja de ruta de Programa Nacional de Productividad y Construcción Sustentable. Santiago de Chile, Chile. Retrieved from https://www.aoa.cl/wpcontent/uploads/2016/03/PMG-Informe-final-Hoja-de-RutaConstruye-2025.pdf

Crane. (2019). Retrieved 26 October 2020, from https://www.3dwasp.com/en/3d-printer-house-crane-wasp/

CyBe Construction. (2020). Retrieved 26 October 2020, from https://cybe.eu/technology/3d-printers/

CyBe. (2012). 3D concrete printers Retrieved July, 2020, from https://cybe.eu/technology/3d-printers

Dini, E. (2019). D-shape. Italy: Dini engineering s.r.I. Retrieved from https://d-shape.com

D-Shape Looks to 3D Print Bridges, a Military Bunker, and Concrete/Metal Mixture - 3DPrint.com | The Voice of 3D Printing / Additive Manufacturing. (2014). Retrieved 26 October 2020, from https://3dprint.com/27229/d-shape-3dprinted-military/

Ghaffar, S. H.; Corker, J. \& Fan, M. (2018). Additive manufacturing technology and its implementation in construction as an ecoinnovative solution. Automation in Construction, 93(October 2017), 1-11.

International Organization for Standardization. (2013). Robots and robotic devices - Coordinate systems and motion nomenclatures (ISO Standard No. 9787:2013). Retrieved from https://www.iso.org/standard/59444.htm

Kazemian A., X. Yuan, E. Cochran, B. Khoshnevis (2017). Cementitious materials for construction-scale 3D printing Laboratory testing of fresh printing mixture, Constr. Build. Mater. 145 639-647.

Kazemian, A., Yuan, X., Meier, R., Cochran, E. \& Khoshnevis, B (2017). Construction-scale 3D printing: Shape stability of fresh printing concrete. Proceedings of the ASME 2017 12th International Manufacturing Science and Engineering Conference, June 4-8, 2017, Los Angeles, CA, USA. 
Khoshnevis, B., Hwang, D., Yao, K. T., \& Yeh, Z. (2006). Megascale fabrication by contour crafting. International Journal of Industrial and Systems Engineering, 1(3), 301-320

Khoshnevis, B., Russell, R., Kwon, H. \& Bukkapatnam, S. (2001). Crafting large prototypes, IEEE Robotics \& Automation Magazine, 8, (3), 33-42.

Lim, S., Buswell, R. A., Le, T. T., Austin, S. A., Gibb, A. G. F. \& Thorpe, T. (2012). Developments in construction-scale additive manufacturing processes. Automation in Construction, 21, (2012), 262-268.

Ma, G. W.; Wang, L. \& Ju, Y. (2018). State-of-the-art of 3D printing technology of cementitious material-An emerging technique for construction. Science China Technological Sciences, 61(4), 475-495.

Perkins, I. \& Skitmore, M. (2015). Three-dimensional printing in the construction industry: A review. International Journal of Construction Management, 15(1), 1-9. $\begin{array}{llll}\text { Putzmeister } \quad \text { (2020). Retrieved } & \text { from }\end{array}$ https://www.putzmeister.com/documents/20152/53599/MT+3 213-10+ES+-+P12.pdf

Véliz-Reyes, A., Jabi, W., Gomaa, M., Chatzivasileiadi, A., Ahmad, L. \& Wardhana, N. (2019). Negotiated matter: a robotic exploration of craft-driven innovation. Architectural Science Review, 62(5), 398-408.

WASP. (2015). BigDelta WASP 12m. Retrieved from https://www.3dwasp.com/en/giant-3d-printer-bigdelta-wasp$12 \mathrm{mt}$

WASP. (2018). Crane WASP. Retrieved from https://www.3dwasp.com/en/3d-printer-house-crane-wasp 